\title{
What Can Windows Learn From Pictures?
}

\author{
JONATHAN LOUIE
}

Syracuse University

\begin{abstract}
What can Windows learn from Pictures? Lately, it seems, the architects concern has been relegated to specifying and locating the Window, and manufacturers imbue Windows with other characteristics, but, for centuries we have understood the Window as a metaphor for media that represent reality, the real, in art. The article highlights windows and projects whose aims are to connect visuality with architecture by reclaiming the view through the window as a collection of material stuff that establish the visual and informational range of the subject beyond; linking seemingly immaterial imagery to the presence of matter.
\end{abstract}

If the aspiration of new technologies is to eventually make hardware disappear, the picture window needs less material stuff to perform. ${ }^{1}$

- Liz Diller and Ricardo Scofidio in conversation with Arthur Lubrow, "Architects, In Theory"

\section{INTRODUCTION}

While preparing for their 2003 mid-career retrospective at the Whitney Museum, Liz Diller and Ricardo Scofidio recounted a story to New York Times Critic Arthur Lubrow on the conception of their unbuilt Slow House. '(In 1989) Our client came to us and said he wanted a house with a view,' the duo stated wryly. Perhaps it was just an innocent request, a simple nod towards the fact that the house needed large picture windows because the property was located along the Long Island waterfront. But given their body of work that speculate on how one views, with titles such as: 'Window Room and Furniture (1981), 'Mirror Gravity Levity (1971), 'The Delay in Glass' (1987), or 'Three Windows' (1986), he must have assumed that Liz and Ric had more to say about Windows. Perhaps then, the clients request alludes to an ongoing disciplinary problem between the Window as a practical device (a material opening for access to light and air), and the Window as an epistemological metaphor (a figure for framing a representation of the subject), each of which lay claims towards the act of creating a view.

In their interview, Diller and Scofidio argued as much, that the Slow Houses' Picture Window represents a more advanced technology than the pictures in the television display -- 'because it strips away the hardware that you have on a TV monitor and leaves only the effect. ${ }^{2}$ But upon further review I think the architects had more to say. After all, by coupling the Picture Window with the monitor, the designers call attention away from the Window frame and towards the Windowpane by stating their consideration for the qualities of the surface material and its' effects. From a broader point of view, the statement seeks to reclaim the intimate relationship between the window and visuality as an architectural project; as architects typically specify the location and type of Windows, but it is primarily in the visual arts that theories on viewing have been advanced. By taking Diller and Scofidios' claim literally, this paper recounts well-known episodes in Western art to connect visuality with architecture by considering the Window as a link between immaterial imagery and the presence of matter.

\section{THE WINDOWFRAMES AS SUBJECT}

The study of the Window as a viewing device, not the study of the subject matter and how it is depicted.

The act of stripping the cathode ray tubes from the television screen may at first seem crude; but to consider the statement as a commentary on creating a view offers useful insight towards understanding the Picture and the Window today. Simply put, what is the trajectory of visuality if the Windowpanes' materiality is the subject of inquiry? While the relationship between television and window may seem obvious, after all the development of the Picture Window in post war American architecture roughly coincided with the distribution of the television imagery into domestic space. ${ }^{3}$ And the material hardware, from pigment to cathode ray tube to cinema screen, constitute the physical stuff that filled the frame and reconfigured the transmission of imagery to the built environment. In contrast, for nearly 500 years prior, the conception of the metaphorical Window referred to an absence to be filled, an 'open' window, it is 'a frame for its variable content, (and) a marker of difference between what is inside and outside." Due to the formal resemblances to a canvas, the window as frame and mullion have been a pivotal trope in debates about the origins, practices, and traditions of perspective; and later its' terms of articulation set the framework for future apparatuses ${ }^{5}$ to construct vastly different conditions for presenting the subject as reality, the real, through art. ${ }^{6}$

Simply put, Diller and Scofidios' textual stripping of the television hardware asks pertinent questions about what constitutes the Windows' effects in visual society today. Is the Window a framework for representation, as seen in the writings of Alberti, or a basis for defining a specific medium as seen in 


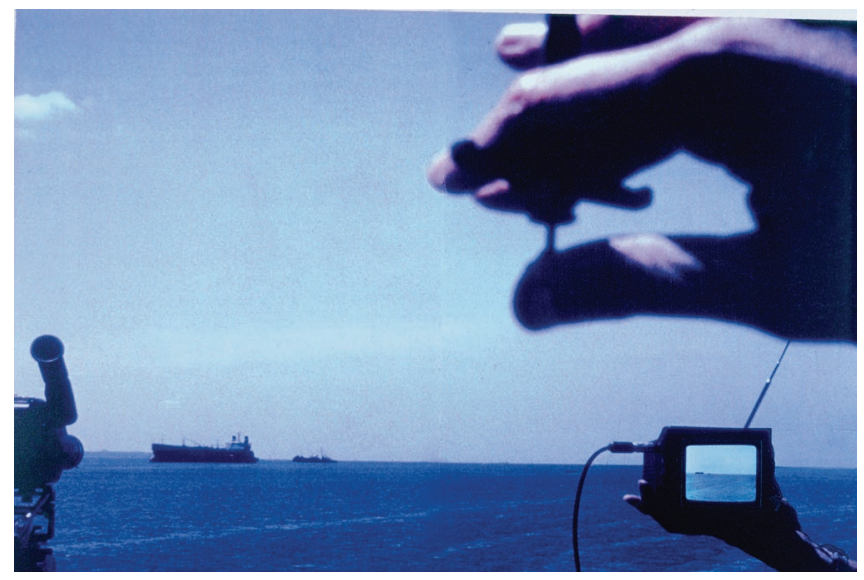

Figure 1. Horizon View, Slow House, Diller and Scofidio + Renfro (1989.)

the articles of Clement Greenberg and Michael Fried? Or, is it what Liz Diller and Ricardo Scofidio suggest by literally building a house of two windows with different 'material stuff,' and creating a view with a myriad of material characteristics and qualities. Flattening, into a single gaze, the Picture emitting from cathode ray tubes alongside a view through the pane of a Window (figure 1).

Nearly thirty years after the Slow House, in a contemporary age where both digital and analogue devices are drastically changing our perception of reality. The sheer number of windows, monitors, and digital screens jumble perceptual qualities with material characteristics. We find ourselves spending more and more of our time staring at the stuff on screens of movies, mirrors, glass, and digital devices - they are windows full of moving images, reflections, graphics, and icons. While these spaces proliferate in another realm or depict another time, they now constitute the appearance of the world by remaining materially located within the 'real space' of hardware. Raising the question: if for over 500 years painting benefitted from windows, what then, can windows learn from pictures?

\section{THE WINDOW PHVEES COMBINES, REALMS.}

The window is affected by a network of physical and conceptual material structures that support its' appearance.

In fifteenth century Florence a typical Florentine window was not visually open or clear but instead filtered and translucent. They were often built of frames stretched with an Impannate a translucent oiled linen that negotiate the movement of light and air. However, unlike the material realities in Renaissance Florence, the conception of the metaphorical window described in De Pictura (1435) voids the windowpane of its' physical characteristics as the foundation for siting a distinct virtual space within the space we occupy. To do so, Alberti instructs painters to not look at the properties of the physical windows around them, but instead, consider the clarity they imagine it to have in their mind.
While Alberti likened the picture plane to an open window to create a conceptual and geometric link between perspective and the terrestrial realm. Leonardo Da Vincis' design for a perspective machine proposes a visual aperture of glass that acts as both substrate and picture plane. He writes: 'Perspective is nothing but seeing a place directly through a completely transparent pane of glass on the surface of which the objects behind the glass are to be drawn. ${ }^{\prime 8}$ Unlike drawing apparatuses that rely on the grid (veil) or geometry (open window) as devices to represent a subject; Da Vincis' machine relies on the ability for the artist to see through a reasonably transparent and textured substrate. The machine is comprised of three elements: the object, the pane of glass, and the eye. The glass pane is placed on a tabletop, where the artifact is viewed with one eye through a small hole in a square frame. One hand steadies the machine and the other hand reaches forward to draw the objects' projection on the vertical glass pane. By marking the surface, Da Vincis' machine indexes an imaginary section, the image, through the cone of vision in real space.

In Da Vincis' machine the transmission of the image - from subject to eye - does not replicate the object but is mediated by the qualities of the glass material. While in concept the glass pane allows for a clear view, in reality the imperfections of spun glass ${ }^{9}$ distort the perception of the subject. Simultaneously influencing perspectival accuracy by both disrupting the view and unsettling the hand. In Renaissance Florence, Da Vincis' pane amounts to a perspectival support that combines visual technique with physical surface to construct a representation that is physically and visually altered by the glass substrate.

\section{THE WINDOW CONSTRUCTS, MEASURABLE EFFECTS VISUAL QUALITIES}

The visual expression(s) of the idea or intent in the work, including: line, shape, color; as well as, material appearance: reflection, texture, and opacity to name a few.

Time and time again Duchamp insisted that the material collections of the 'The Bride Stripped Bare by her Bachelors, Even' (The Large Glass) amounted to a study in perspective. And his notes in the Green Box support his interest in carefully plotting points along a flat plane that appear as a figure in recession. In concept this procedure considered the picture plane as the point of intersection, between the eye of the observer and the imaginary object beyond. In reality, the materiality of the glass results in the oscillation between the appearance of an object and apparition of a perspectival figure. At just under nine feet high and five and a half feet wide, and freestanding within aluminum supports The Large Glass (figure 2) dominates your view. It is too large to take in with one glimpse. Your eyes travel over it randomly: you look at it and through it, you see other viewers moving and stopping, the reflection of the 


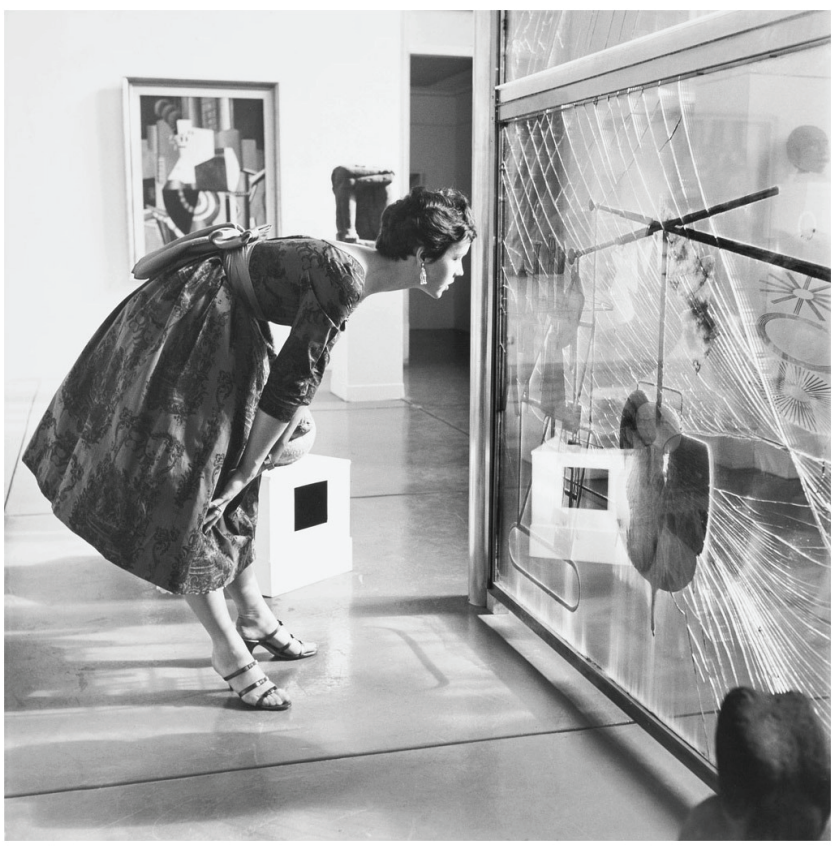

Figure 2. The Large Glass, Photograph by Hermann Landschoff (1954).

lights on its surface, and its mechanical figures on display. The qualities of the image flicker as your eyes move around the piece. To art historian Jean Clair, the 'painting vanishes, (as) the readymade objects make their appearance, ${ }^{10}$ swerving the viewers recognition of the work from painting to sculpture, and from iconographic figures to manufactured objects; the window constructs an assembly of perspectives without the use of traditional painterly techniques.

By using an analogy to the Arms of Saint-Etienne ${ }^{11}$ (the early twentieth-century French equivalent of a Sears and Roebuck catalog), Duchamps' written notes underline the encyclopedic character of The Large Glass as a surface for ideas and things to be collected and displayed. Duchamp even bred dust by taking the glass pane and placing it on sawhorses by an open window, exposing it for a year to the grit of the streets of New York City below. While most of the dust was eventually wiped clean, a few select patches were left untouched to color his composition. Resulting in chance encounters between material qualities that could not have been predicted. Unlike the picture plane that sites another realm within our own, to Duchamp, The Large Glass is a 'hilarious picture,' a window whose surface exhibits material effects with qualities of all kinds.

Unlike the pictorial formalism that dominated the early 20th century, The Large Glass cannot be classified through techniques derived from cubist painting and gestalt psychology. By sandwiching the qualities of manufactured materials between the glass panes Duchamps' work foreshadows a shift from classical painting to modern materials to contemporary media by describing a specific condition, the translation of the windows' pictorial qualities (through the visual arts) back onto itself. ${ }^{12}$ Duchamps' turn towards the Window in the early 20th century was a mediation upon material and technique as a means for innovating upon traditional painting. Its' appearance is akin to Vitrine, a showcase behind a Picture Window, that displays an assortment of material experiments within its' glass panes. While the individual experiments are not readily classifiable as art, the myriad of materials and their visual qualities establish the visual and informational range of the view beyond.

\section{WINDOW,-SPACE-DEPTH}

The existence of distance in a windows substrate portrays the appearance of: dimension, time, and location, that affects the reading of its qualities.

Images saturate contemporary culture; they are illuminated through pixels, reflected off glass, and constantly recurring in photographic reproductions. In a contemporary age where both digital and analogue windows are influencing our view of reality: how do you make a material you have never seen?

Materials.jpg is an image research project by the author that develops machine protocols to produce physical materials through the digital manipulation of Texture Maps (figure 4). The project is equal parts process (the transmission of image data) and product, and constructs images that appear to be but are not the original thing itself (neither original building, or even the first digital image). Unlike replicas or copies, the image-objects approximate the appearance of real materials that exist on the screen but no longer maintain a counterpart in the physical world. Resulting in a presence that is simultaneously appearing to reveal the systems of logics that alter the image, as well as, act as an identifiable link to a familiar thing (a material, object, or building in another place and time). Resulting in something that, according to Anne Friedberg, is a virtual approximation of reality: it appears to be, but is not, of the same materiality as what it represents. ${ }^{14}$ Connecting visuality with architecture by considering the Window as a link between immaterial imagery and the presence of matter.

Like Duchamps 'Large Glass,' that displays visual experiments built from readily available materials, Materials.jpg is built from a collection of materials - petg, ink, and paint - that work together to construct a view. When looking through the frame viewers see recognizable traces of a low-resolution Texture Map (JPEG) that depict a building material whose appearance displays traces of the transmission and translation of its' data: the pixels of the diffuse map, the surface geometries of the height map, and the passing routes the CNC mill. Yet, while the physical depth is shaped by transmission of image data, the windowpanes' range of effects cannot be attributable to a single entity. The result is physical matter whose appearance strays from the first Texture Map to reveal the layers of software protocols that mediate a digital image today. 


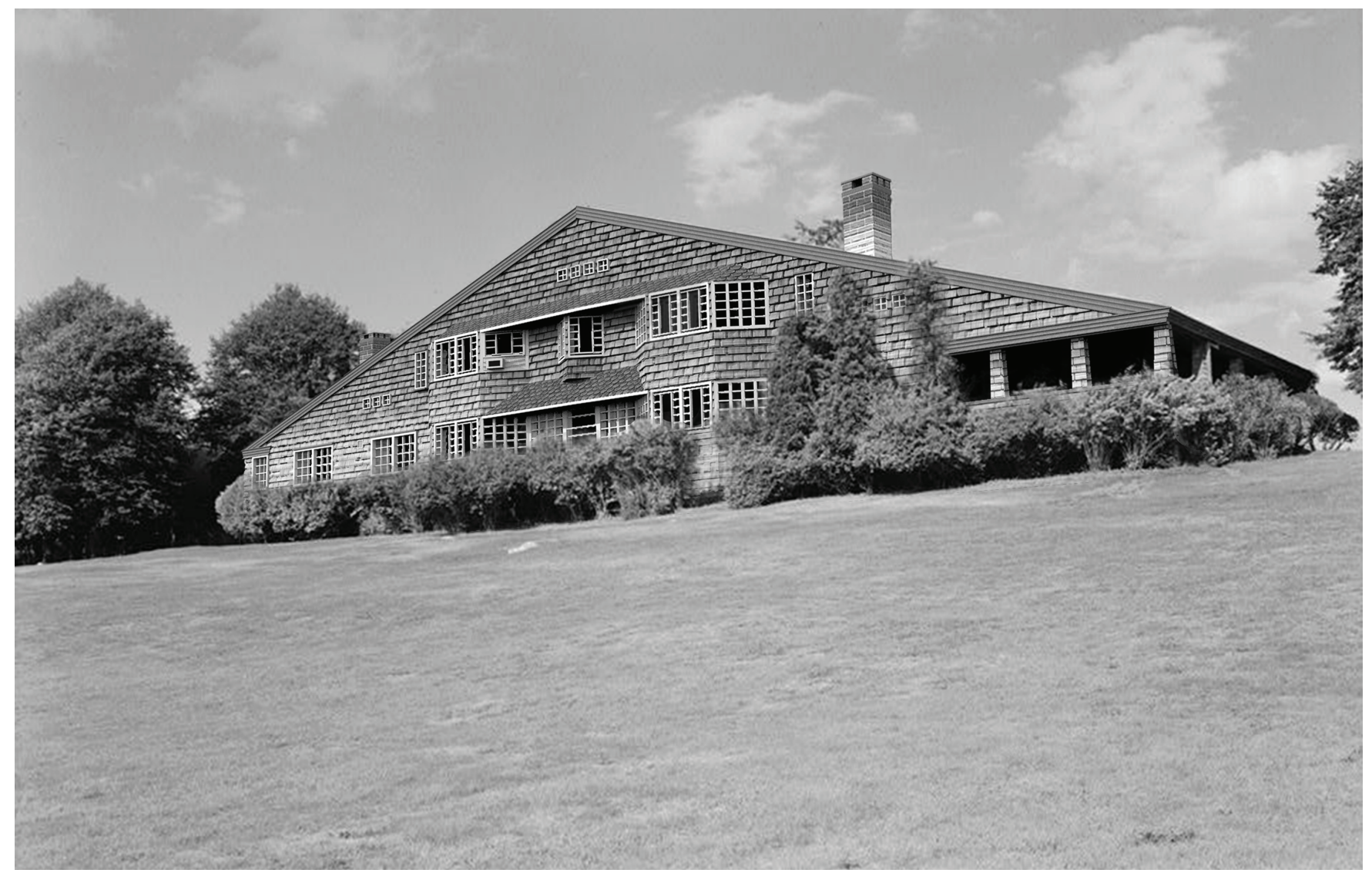

Figure 3. The William G. Low House (demo. 1962, Mckim Mead \& White) reconstructed with Texture Maps, Courtesy of the Author

While Albertis' window to the world constructed a perspectival system for developing an image of the world with reasonable fidelity. In Computer Science, the Texture Maps is as an image wrapping software technique for projecting surface texture and color information that approximates the appearance of stuff in the world - material, graphics, icons, or other. Its' invention in 1974, as a doctoral project by Edwin Catmull, was as a method for mapping photographs 'onto patches thus providing a means of putting texture onto computer generated pictures. ${ }^{15}$ On one hand, Catmulls' research was a basis for controlling an environmental simulation shading and texture - on surfaces and curved solid objects in digital space. On the other, as a signifier, the Texture Map appears as a physical entity whose origins are in another location and time. For example, the application of Texture Maps (and more complex multi-map rendering packages) simulate near-photoreal scenes that foreshadow buildings to come (ie.in the hands of professional rendering offices), as well as, engage the physical world as material approximations for the reconstruction of physical artifacts that are lost (in this instance the William G. Low House by Mckim Mead and White, figure 3). In each instance the act of wrapping a Texture Map atop a model signifies a link between two realms that transverse space and time.
Despite their familiar appearance, the Texture Maps should not be classified as faithful reproductions. Materials.jpg challenges the classical Albertian relationship of the image as a reflection on reality, to now constructing a new reality in of itself. In a world saturated by images Materials.jpg are simultaneously object and image, and their characteristics reflect qualities of both.

\section{CONCLUSION}

Nearly thirty years after the conception of the Slow House, to reconsider Diller and Scofidios' verbal stripping of the monitor hardware signals a shift in how architects engage the limits of the visual environment. In a world increasingly saturated by Pictures the Window no longer solely defines a subject through the mediums of drawing and painting; and the view through the window is no longer a representation of the physical world. While these pictures and spaces proliferate in another realm or depict another time, they now alter the appearance of the world by being materially located within hardware. From the rippled glass of Da Vincis' machine, to the material collections of Duchamp, and the translation of digital image in Materials.jpg, when Windows learn from Pictures both systems of representation and material are in productive exchange. 

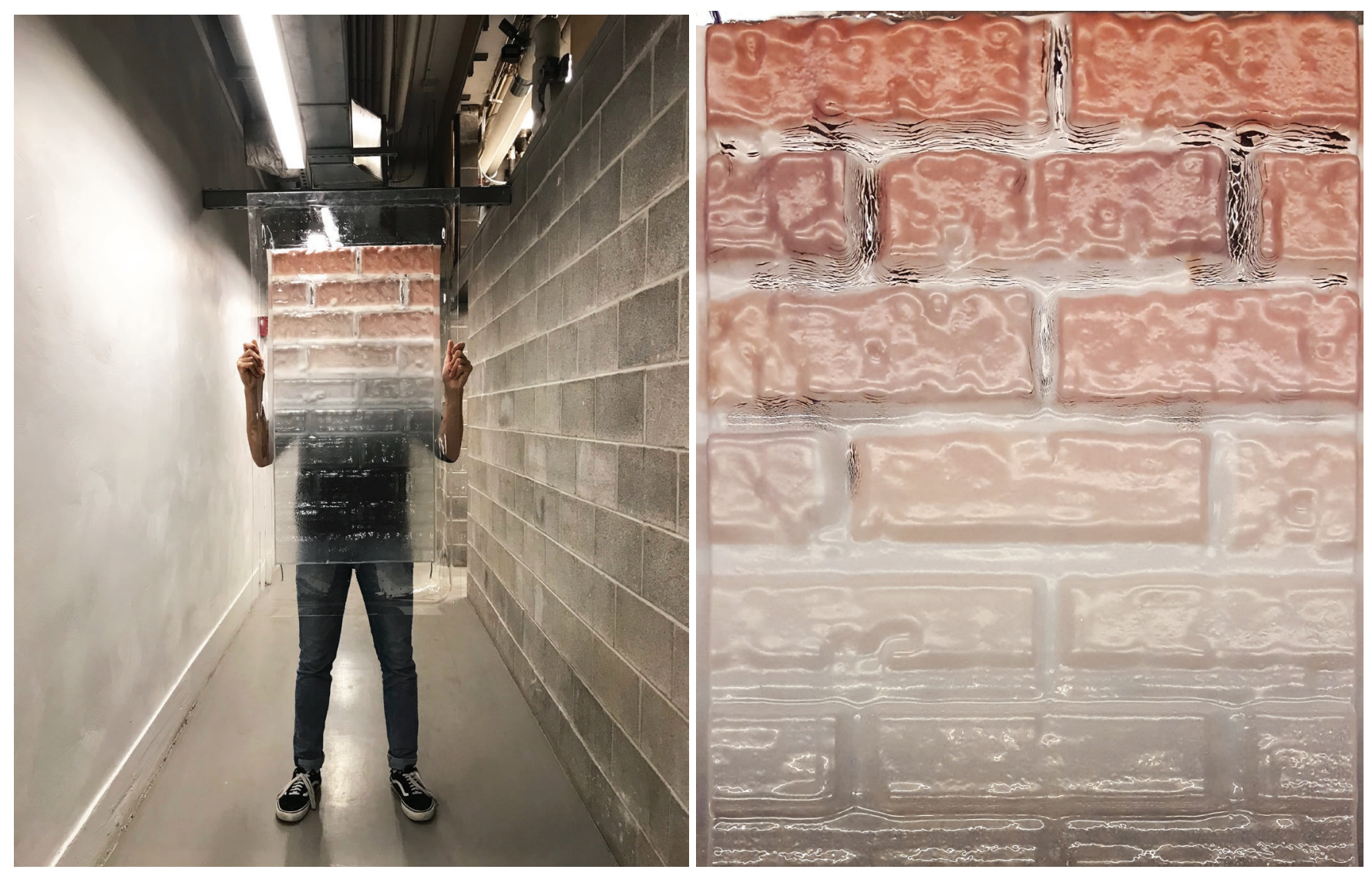

Figure 4. Brick Windowpane Mock-Up, one of a series that comprise windows that construct links to the William G. Low House, Courtesy of the Author.

\section{ENDNOTES}

1 Arthur Lubrow, "Architects, In Theory," New York Times, February 16, 2003. https://www.nytimes.com/2003/02/16/magazine/architects-in-theory.html.

2 Lubrow, "Architects, In Theory."

3 Lynn Spigel, Make Room for TV: Television and the Family Ideal in Postwar America (Chicago; London: The University of Chicago Press, 1992), 102.

4 Julia Orwell, "Window," Theories of Media, accessed December 22, 2018. http:// csmt.uchicago.edu/glossary2004/window.htm\#_edn3.

5 For example, at the Slow House the glazing of a picture window acts to simultaneously portray surface and space by drawing attention to the reflective surface of the glazing. On the other hand, the TV monitor depicts the ocean view by projecting a sequence of flickering cathode ray tubes.

6 Hal Foster, Return of the Real: Art and Theory at the End of the Century (Cambridge, MA: The MIT Press, 1996). To Hal Foster, ideas borne in painting (specifically Pop Art) are an unbroken continuation of ideas that drove the earlie avante-grades. Linking the development of present ideas as part of a continuous tradition for painters to consistently pursue their 'area of competence.'

7 Elizabeth Grosz, Architecture from the Outside: Essays on Virtual and Real Space (Cambridge, MA: The MIT Press, 2001), 40.

8 J.P Ricther, The Literary Works of Leonardo da Vinci, third edition, (New York: Phaidon, 1970), 150.

9 While the common use of blown and spun glass windows was increasingly available in the 18th and 19th century. See "The Crystal Palace" (1851) or other Greenhouses by Joseph Paxton. The mass consumption of the picture window relied on the development of float glass technologies by Alastair Pilkington in the 1950s.

10 Jean Clair, "Duchamp and the Classical Perspectivists," Artforum 7 (1978): 47.

11 Pierre Cabanne,"Unpacking Duchamp," Dialogues with Duchamp (Cambridge, MA: Da Capo Press, 1987), 42.

12 Mark Linder, "Architecture More or Less," in Beyond Surface Appeal: Literalism, Sensibilities, and Constituencies in the Work of James Carpenter, ed., Sarah Whiting (Cambridge, MA: Harvard Graduate School of Design, 2012). For my interest in the overlap of art and architecture through Pop and Literalism in the 1950s, 1960s, 1970s, I am indebted to texts by Mark Linder and Hal Foster. In particular, this segment references Mark Linders's writing that situates James Carpenter's architectural installations in "Architecture More or Less."

13 Marcel Duchamp, "The Brides Veil," in Salt Seller, the Writings of Marcel Duchamp, eds., Michel Sanouillet and Elmer Peterson (London: Oxford University Press, 1974). Using the analogy to the Arms of Saint-Etienne (the early twentieth-century French equivalent of a Sears and Roebuck catalog), Duchamp underlines the encyclopedic character of this work that are collected and displayed. Duchamp considers the relationship between The Large Glass and the Shop Window as two modern innovations. In his subsequent notes Duchamp considers the role of the glass panes as a show case for the placement of fragile objects inside.

14 Anne Friedberg, "Introduction: The Virtual Window," in The Virtual Window (Boston: The MIT Press, 2009), 8.

15 Edward Catmull, "A Subdivision Algorithm for Computer Display of Curved Surfaces," PhD diss., University of Utah, 1974. 